\title{
Wavelet based Fault Classification for Rolling Element Bearing in Induction Machine
}

\author{
Amit Shrivastava \\ Research Scholar, Department of Electrical \\ Engineering Madhav Institute of Technology \& \\ Science, Gwalior, M.P
}

\begin{abstract}
Induction motors plays the most important role in any industry. Induction motor faults results in motor failure causing breakdown and great loss of production due to shutdown of industry and also increases the running cost of machine with reduction in efficiency. This needs for early detection of fault with diagnosis of its root cause. In this research paper a wavelet based fault classification method has been developed for rolling element bearing in induction motor using vibration signal. Wavelet based vibration analysis is one of the most successful techniques used for condition monitoring of rotating machines. This paper describes a new condition monitoring method for induction motors based on wavelet transform. A robust bearing fault detection scheme has been developed by time-frequency domain feature extraction from vibration signals of healthy and defective machine.
\end{abstract}

\section{General Terms}

Induction motor, fault detection, ball bearing, wavelet transform, condition monitoring.

\section{Keywords}

Induction motor, fault classification, condition monitoring, rolling element bearing, wavelet transform.

\section{INTRODUCTION}

In this paper, signals obtained from monitoring system are treated with different processing techniques with suitably modified algorithms to extract detailed information for machine diagnosis $[1,2]$. A brief review about signal processing and analysis techniques with their implementation on the data obtained from the machine under test is presented here. The result of this investigation demonstrates the usability and ability of the proposed methods for predicting the machine faults.

The vibration signals are recorded for healthy as well as defective bearing condition. The acquired vibration signals are then analyzed in time-frequency domain.

\section{SIGNAL ANALYSIS}

A simple approach for estimating the condition of a machine is to measure the overall vibration signal intensity and to compare this with the reference value corresponding to healthy machine. Other approaches are based on signal analysis in time and frequency domain [3, 4]. The time domain analysis provides information about the signal whereas frequency domain analysis provides detailed information on frequency components of the signal $[5,6]$.

\author{
Sulochana Wadhwani, Ph.D \\ Associate Professor,Department of Electrical \\ Engineering Madhav Institute of Technology \& \\ Science, Gwalior, M.P
}

\section{FEATURE EXTRACTION USING WAVELET PACKET TRANSFORM (WPT)}

Wavelet transform is used here for extracting important features from the recorded vibration signal. For wavelet packet decomposition up to level ' $\mathrm{J}$ ', the original signal is described by (2J+1-2) wavelet packet coefficient vectors or nodes. By employing suitable threshold, those wavelet coefficient of given decomposition vector, which in less than the set threshold can be neglected. Therefore, the wavelet transform can present the signal with limited number of coefficients. These coefficients can directly be used as the fault. However, to obtain the signal numerical measure of the decomposition vector, RMS value of the node, which is the measure of the signal intensity contained in specific frequency band, can be used more conveniently.

In this strategy, RMS value at each wavelet packet node is defined as an individual feature component and is used as a robust rudimentary exploration of the specific signal features that provide useful information for classification purpose. The RMS value is computed for twenty eight nodes. A total of one hundred vibration signals, twenty five from each fault case are taken for computation level. The mean and standard deviation of the obtained rms value for each fault is obtained for a given node and are shown in figure 1.

\section{REDUCTION IN FEATURE VECTOR}

Main objective of this work is to find those decomposition vectors/nodes of the vibration signal, which can best describe the fault condition. Reduction in feature vector dimensionality can be achieved by selecting only those components, which have maximum class separability information. To find the most representative set of the features of each bearing state, equation 1 is used for ability of feature ' $F$ ' to discriminate between case $A$ and case $B$

$$
\gamma_{A-B}(F)=\frac{M_{A}(F)-M_{B}(F)}{\sigma_{A}(F)+\sigma_{B}(F)}
$$

The pairs of classes are formed corresponding to three bearing defect: defective balls, defective inner race and defective outer race with healthy bearing condition. The results of the Figure 1.are used for computation of coefficients $\Upsilon_{\mathrm{A}-\mathrm{B}}$. Before identifying those nodes, which carry significant information, YA-B coefficients are normalized by using Equation 2 given below:

$$
\text { Normalized value } \gamma_{A B}=
$$

$$
\frac{\text { actual value of } \Upsilon_{A B}-\text { minimum value of } \Upsilon_{A B}}{\text { maximum } Y_{A B}-\text { minimum } Y_{A B}}
$$




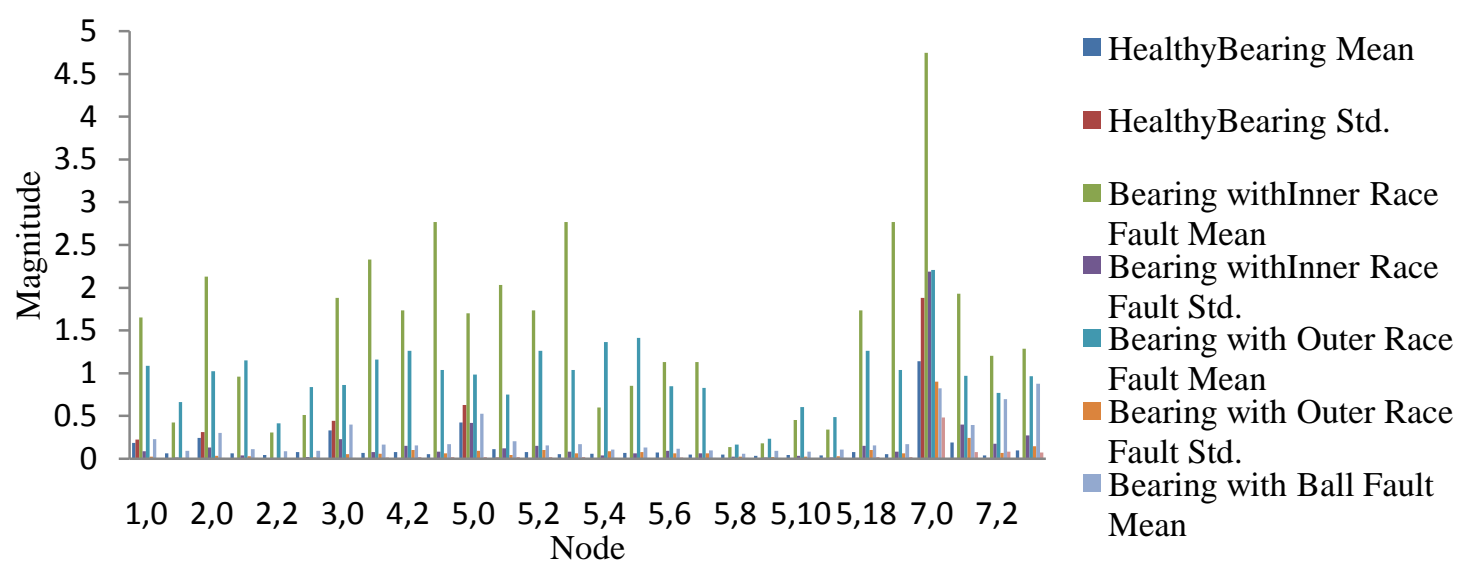

Fig 1:RMS Values of Vibration at Various Nodes

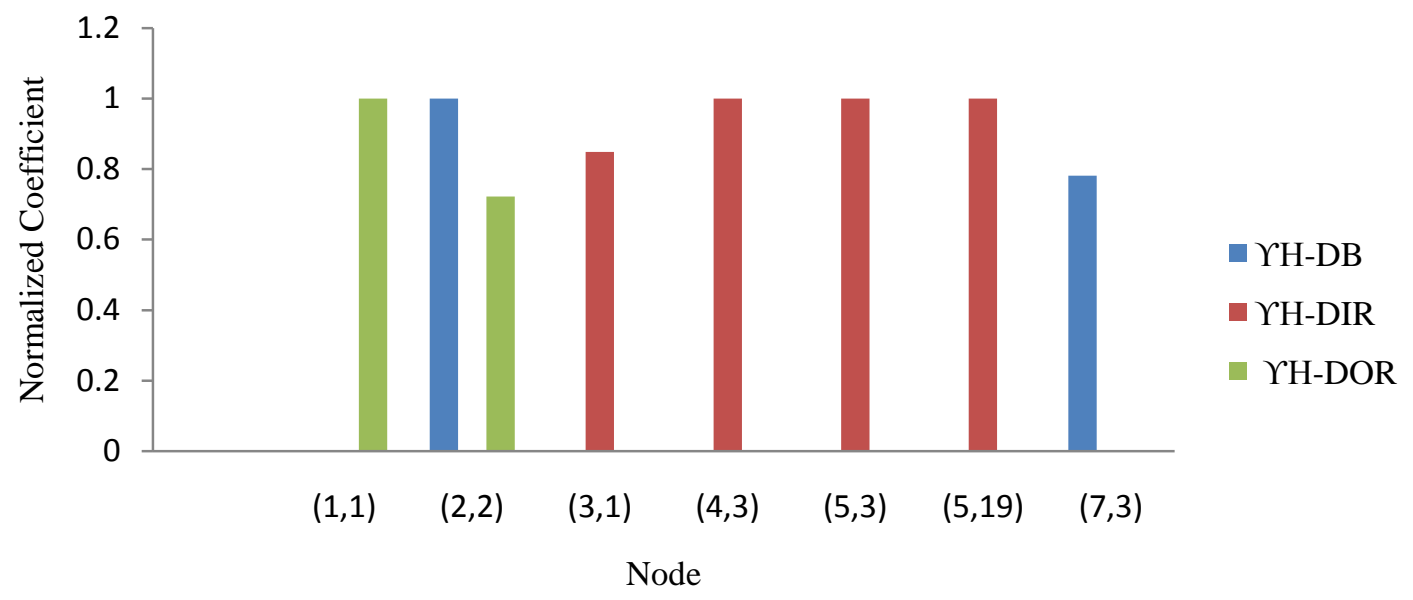

Fig. 2: Normalized YA-B for H-DB, H-DIR, H-DOR

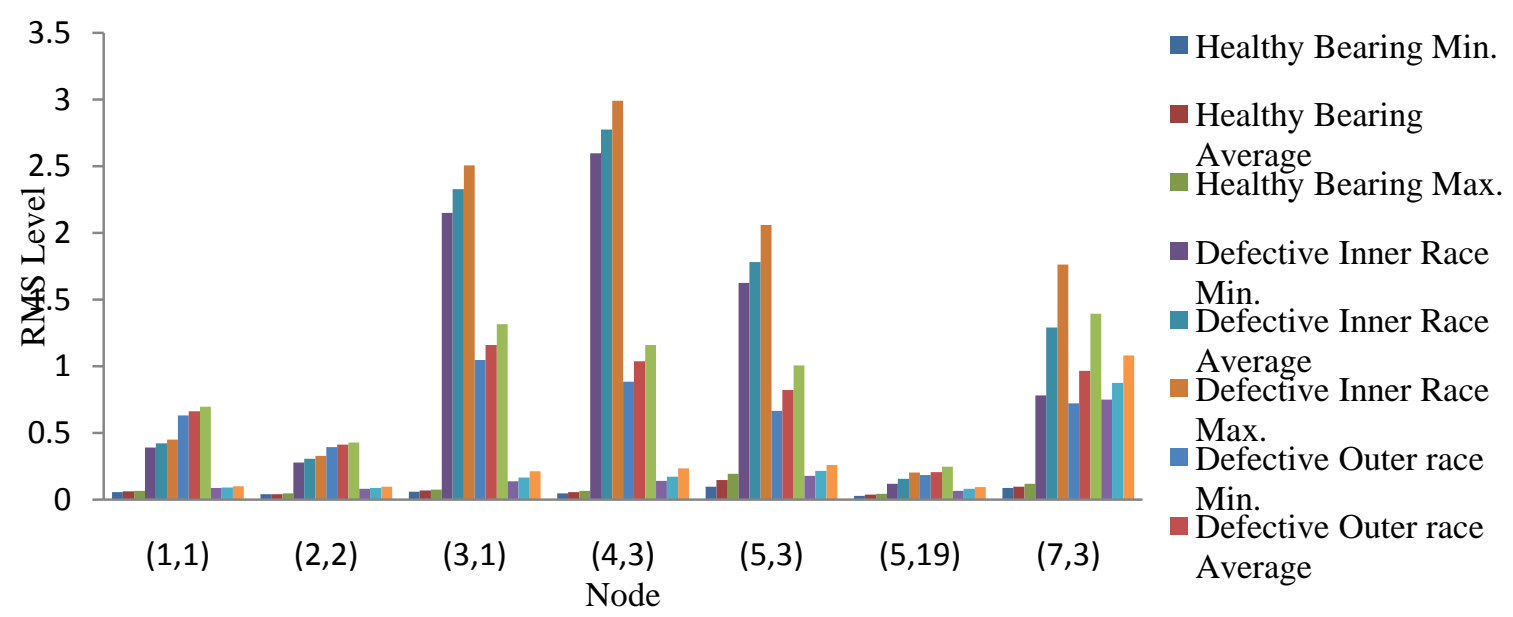

Fig. 3: RMS Values of Vibration at Specified Node 
This information brings the coefficient for each in the range of $0-1$. The nodes for which normalized coefficient is greater or equal to 0.7 are identified for each class pair and are shown in Figure 2.

Figure 2. gives winner nodes for class pair healthy bearing and defective ball, healthy bearing and defective inner race, healthy bearing and defective outer race. From the findings given in Figure 2 nodes shown in Figure 3 are selected as fault features. In this way total twenty eight features are reduced to seven wavelet node RMS features. The RMS value corresponds to frequency $1-1.5 \mathrm{kHz}$ has highest class discriminate coefficient, followed by RMS value of frequency $0.0468-0.0625 \mathrm{kHz}$. In case of defective inner race, the RMS value of node having frequency band of $0.3750-0.5000 \mathrm{kHz}$ has maximum fault information, followed by $0.1875-0.2500$ $\mathrm{kHz}, 1.1875-1.2500 \mathrm{kHz}$ and $0.2500-0.5000 \mathrm{kHz}$. In case of defective outer race, the RMS value of node having frequency band of 1-2 kHz has maximum fault classification information followed by $1.0000-1.5000 \mathrm{kHz}$ as shown in Figure 3 .

\section{RESULTS AND DISCUSSIONS}

Wavelet Packet Decomposition was applied on 30 data set of healthy bearing, Inner race fault, outer race fault and defective ball up to eight levels resulting in 510 nodes. The minimum, mean and maximum RMS values of vibration of decomposition vector corresponding to these seven nodes are obtained for each bearing fault case. Further, normalization was done on all 510 nodes. After normalization it is found that the nodes for which normalization coefficient is 0.7 give good classification of faults. On the basis of which seven nodes namely $(1,1),(2,2),(3,1),(4,3),(5,3),(5,19)$ and $(7$, 3 ) were selected. The variation of the RMS value for healthy bearing, defective ball defective inner race and defective outer race at the selected seven nodes $(1,1),(2,2),(3,1),(4,3),(5$, $3),(5,19)$ and $(7,3)$ were plotted and are shown in Figure 3. Node $(7,3)$ can classify the condition of fault. It shows that if the RMS value at this node is greater than 0.6 then there is a fault in bearing of motor. But this node do not gives the information for identification of fault type. From Figure 3 it can be clearly seen that rms value of decomposition vector for nodes $(1,1),(2,2),(5,3)$ and $(5,19)$ is having very close values for different defects and thus cannot differentiate between race defects. These nodes are hence dropped from the final feature set. Node $(3,1),(4,3)$ and $(5,3)$ gives good classification between defective inner race and defective outer race, defective inner race and defective ball, defective outer race and defective ball. Thus these three nodes were selected for different fault classification.

\section{REFERENCES}

[1] Renwick J.T. 1984 Condition Monitoring of Machinery Using Computerised Vibration Signature Analysis. IEEE Trans. On Industry Applications, 519-527.

[2] Thomson W. T., 2001 Current Signature Analysis to Detect Induction Motor Faults, IEEE Industry Applications Magazine, (July/Aug. 2001), 26-34.

[3] Kliman G. B., Premerland W. J., Koegl R. A., and Hoeweler D., 1996 A New Approach to On-Line Turn Fault Detection in AC Motors, in Conf. Proec. IEEE IAS'96, Vol. 1, San Diego, CA, USA, 687-693.

[4] Altmann J., Mathew J., 2001 Multiple Band-Pass Autoregressive Demodulation for Rolling Element Bearing Fault Diagnosis, Mechanical Systems and Signal Processing, Vol 15, No.5, 963-977.

[5] Tse P.W., Peng Y. H., Yam Richard, July 2001 Wavelet Analysis and Envelope Detection for Rolling Element Bearing Fault Diagnosis: Their Effectiveness and Flexibilities, Journal of Vibration and Acoustics, Vol. 123, No.3, pp 303-310.

[6] Silva A.A. Da et al.,(1997) Rotating Machinery Monitoring and Diagnosis using Short Time Fourier Transform and Wavelet Techniques, in Proc. International Conference Maintenance \& Reliability, Vol. 1, Knowville, TN, 14.01-14.15.

[7] Shrivastava Amit, Wadhwani Sulochana Vibration Signature Analysis for Ball Bearing of Three Phase Induction Motor, International Journal of Electrical and Electronics Engineering (IOSRJEEE). Volume 1, Issue 3, July-August 2012, 46-50. 